\title{
Volume of distribution and clearance of peptide-based nanofiber after convection-enhanced delivery
}

\author{
Ranjodh Singh, BPhil, BSc, ${ }^{1}$ Vanessa Bellat, PhD, ${ }^{2}$ Melinda Wang, BA, ${ }^{1}$ \\ Melanie E. Schweitzer, BSc, ${ }^{1}$ Y. Linda Wu, BA, ${ }^{1}$ Ching-Hsuan Tung, PhD, ${ }^{2}$ \\ Mark M. Souweidane, MD, ${ }^{1,3}$ and Benedict Law, PhD²
}

\author{
${ }^{1}$ Department of Neurological Surgery, ${ }^{2}$ Department of Radiology, Molecular Imaging Innovations Institute, and ${ }^{3}$ Department of \\ Pediatrics, Weill Cornell Medicine, New York, New York
}

OBJECTIVE Drug clearance may be a limiting factor in the clinical application of convection-enhanced delivery (CED). Peptide-based nanofibers (NFPs) have a high aspect ratio, and NFPs loaded with drugs could potentially maintain effective drug concentrations for an extended period sufficient for cancer therapy. The objective of this study was to assess the volume of distribution $(\mathrm{Vd})$ and clearance of variable lengths of NFPs when administered using CED.

METHODS NFPs composed of multiple methoxypolyethylene glycol (mPEG)-conjugated constructs ( $\mathrm{mPEG}_{2000^{-}}$ KLDLKLDLKLDL-K(FITC)-CONH ${ }_{2}$, for which FITC is fluorescein isothiocyanate) were assembled in an aqueous buffer. The NFPs were approximately $5 \mathrm{~nm}$ in width and were formulated into different lengths: $100 \mathrm{~nm}$ (NFP-100), $400 \mathrm{~nm}$ (NFP-400), and $1000 \mathrm{~nm}$ (NFP-1000). The NFP surface was covalently conjugated with multiple Cy5.5 fluorophores as the optical reporters to track the post-CED distribution. Forty-two 6- to 8-week-old Ntv-a;p53 ${ }^{\text {trf }}$ mice underwent CED to the striatum. Animals were killed immediately, 24 hours or 72 hours after CED. The brains were extracted and sectioned for assessing NFP Vd to volume of infusion (Vi) ratio, and clearance using fluorescence microscopy.

RESULTS CED of NFPs was well tolerated by all the animals. The average Vd/Vi ratios for NFP-100, NFP-400, NFP1000 , and unconjugated positive control (free Cy5.5) were 1.87, 2.47, 1.07, and 3.0, respectively, which were statistically different $(p=0.003)$. The percentages remaining of the original infusion volume at 24 hours for NFP-100, -400 , and -1000 were $40 \%, 90 \%$, and $74 \%$, respectively. The percentages remaining at 72 hours for NFP-100, -400 , and -1000 were $15 \%, 30 \%$, and $46 \%$, respectively. Unconjugated Cy5.5 was not detected at 24 or 72 hours after CED.

CONCLUSIONS CED of NFPs is feasible with Vd/Vi ratios and clearance rates comparable to other nanocarriers. Of the 3 NFPs, NFP-400 appears to provide the best distribution and slowest clearance after 24 hours. NFP provides a dynamic theranostic platform, with the potential to deliver clinically efficacious drug payload to brain tumor after CED. https://thejns.org/doi/abs/10.3171/2017.2.JNS162273

KEY WORDS convection-enhanced delivery; peptide-based nanofiber; mouse; drug clearance; glioma; oncology

$\mathrm{D}$ IFFUSE intrinsic pontine gliomas (DIPGs) are inoperable and lethal high-grade gliomas that account for $75 \%-80 \%$ of pediatric brainstem gliomas. ${ }^{20}$ Despite numerous efforts, median survival is shorter than 1 year. Convection-enhanced delivery (CED) is a local drug delivery technique that bypasses the blood-brain barrier and delivers large and small molecules to the injection site, ${ }^{13,18}$ and has recently shown promise as an efficient drug-delivery method in DIPG treatment. ${ }^{3,7,31}$ CED allows for enhanced localized drug distribution and reduced systemic exposure to patients. ${ }^{30}$
Clearance of inert molecules, such as contrast agents, from the brain interstitium is rapid once delivered by CED. Whether these time constraints would hold true for biologically active drugs remains unknown. Regardless, longer exposure of an antineoplastic agent within a target tissue would be clinically beneficial. Potential ways to circumvent rapid drug clearance after CED include prolonged CED infusions and using drug vehicles to slow clearance. Previously, our group has published research on the feasibility and safety of prolonged CED in naïve animal brainstem. ${ }^{21,35}$ Other experimental studies have also shown long- 
term infusions to be promising therapeutically in brain tumor treatment. ${ }^{6,14,32,36,37,47}$ While prolonged CED is appealing, its technical and safety aspects remain challenging. Longer infusions require designing easily accessible and reusable implantable catheters that can be secured in place and readjusted, if needed. Implantable catheters may increase the risk for infection and mechanical injury from catheter movement. Furthermore, constant monitoring for drug toxicity and volume of distribution $(\mathrm{Vd})$ is required to ensure that prolonged infusions do not lead to systemic toxicity. Despite the advantages of prolonged CED and the recent development of CED implantable catheters, ${ }^{6,11}$ single-session CED remains the current clinical practice.

Drug vehicles, namely nanocarriers, provide another means to circumvent rapid drug clearance after CED. Peptide-based nanofibers (NFPs) loaded with drugs may allow for sustained drug release and maintain effective drug concentrations for an extended period sufficient for cancer therapy. In the current study, we investigated the $\mathrm{Vd}$ and clearance of variable lengths of NFP after CED. We hypothesized that shorter-length NFP would have the largest $\mathrm{Vd}$ and the fastest clearance compared with larger ones.

\section{Methods}

\section{NFP Assembly and Characterization}

NFPs were prepared using the solvent evaporation method previously described. ${ }^{34,52}$ Briefly, fluorescein isothiocyanate (FITC)-conjugated peptide $\left(\mathrm{mPEG}_{2000}-\mathrm{KLDL}\right.$ KLDLKLDL-K(FITC)-CONH ${ }_{2}$, in which $\mathrm{mPEG}_{2000}$ is methoxypolyethylene glycol of $2000 \mathrm{Da}, \mathrm{K}$ is lysine, $\mathrm{L}$ is leucine, and D is aspartic acid) constructs $(0.5 \mathrm{mg})$ in dimethyl sulfoxide $(10 \mu \mathrm{l})$ were added to a cosolvent of acetonitrile and water $(1 \mathrm{ml})$. After 16 hours of reaction, the assembled NFPs were incubated 30 minutes with Cy5.5 NHS ester $(0.1 \mathrm{mg})$. To remove free peptides and unconjugated dyes, NFPs were purified by size-exclusion chromatography (Sephadex G-25) in phosphate-buffered saline (PBS), and then homogenized into 3 different lengths: $1000 \mathrm{~nm}, 400 \mathrm{~nm}$, and $100 \mathrm{~nm}$ using a mini-extruder (Avanti Polar Lipids) and polycarbonate membranes of the appropriate pore size (Whatman). The concentrations of nanofibers and Cy5.5 conjugated on the NFP surface were determined by ultraviolet absorbance according to the extinction coefficient of FITC $\left(60,000 \mathrm{~cm}^{-1} \mathrm{M}^{-1}\right)$ and Cy5.5 $\left(209,000 \mathrm{~cm}^{-1} \mathrm{M}^{-1}\right)$, respectively, in 5\% (v/v) PBS in methanol. NFP morphology and size were characterized using transmission electron microscopy (TEM; JEM-1400 LaB6, JEOL USA, Inc.). Prior to TEM imaging, NFPs in PBS $(20 \mu \mathrm{l})$ were transferred onto Formvar/carbon-coated 400-mesh copper grids (Electron Microscopy Sciences) and the samples were stained with $0.5 \%(\mathrm{v} / \mathrm{v})$ uranyl formate solution. The cytotoxicity of NFPs was assessed using the CellTiter-Glo Luminescent Cell Viability assay (Promega). Brain and brain cancer cells (astrocytes, U87, GS-9 L, and C6 from ATCC) were seeded on a 96-well plate $\left(2 \times 10^{3} /\right.$ well $)$ overnight. The culture medium was then replaced with fresh medium containing different concentrations of NFPs (from $1 \mathrm{nM}$ to $10 \mu \mathrm{M}$, according to the peptide concentration). After 3 days of incubation, the cell viability was measured following the manufacturer instructions. All experiments were performed in triplicate, and the results were presented as mean \pm standard deviation.

\section{Experimental Groups}

This study was approved by the Institutional Animal Care and Use Committee of Weill Cornell Medicine and was conducted according to the Institute for Laboratory Animal Research guidelines. To determine NFP Vd/volume of infusion ( $\mathrm{Vi}$ ) ratio, 14 6- to 8 -week-old Ntv-a;p53 $3^{\text {ft/fl }}$ mice were used. ${ }^{8}$ Two breeding pairs of these mice were obtained from Dr. Oren Becher, Northwestern University, and an in-house colony was established through successive breeding. We tested the NFP in naïve animals of this strain with the eventual intent of testing the NFP in tumor-bearing animals of this strain in future experiments. Animals were divided into 3 experimental groups and 1 control group (Table 1). Group 1 received NFP-100, Group 2 received NFP-400, and Group 3 received NFP-1000. The control group was infused with unconjugated Cy5.5 dissolved in PBS.

Our preliminary studies revealed rapid clearance $(<24$ hours) of small molecules from the brain interstitium after CED (unpublished data, 2014). Other reports show that Vd of Gd-diethylenetriamine pentaacetic acid begins to decrease as quickly as 30 minutes after CED, indicating rapid clearance. Even for large-molecular-weight agents, such as iopamidol, where clearance may be slower, no agent is observable at 72 hours after infusion. ${ }^{4,16}$ We therefore assessed clearance at 24 and 72 hours after CED. Twentyeight 6- to 8-week-old Ntv-a;p53 ${ }^{\mathrm{f} / \mathrm{fl}}$ mice were divided into 3 experimental groups and 1 control group (Table 2). Four animals in each experimental group were killed at 24 hours and the remaining 4 at 72 hours. Each experimental group received a different length NFP, while the control group only received unconjugated Cy5.5 dye.

\section{CED Procedure}

The CED procedure in mice has been previously described ${ }^{57}$ Briefly, animals were anesthetized using isoflurane and placed in a stereotactic frame (Model 900; David Kopf Instruments). A midline 5-mm scalp incision was made, and the animal's skull was leveled using bregma and lambda as reference points. A bur hole $0.2-\mathrm{mm}$ in diameter was made $0.5 \mathrm{~mm}$ anterior to bregma and $2.5 \mathrm{~mm}$ lateral to the right of midline. A 33-gauge blunt needle with a Nanofil syringe (World Precision Instruments) mounted on a UMP-2 pump (World Precision Instruments) was lowered vertically to a depth of $3.5 \mathrm{~mm}$ below the skull surface to target the striatum. Prior to insertion, the needles were primed with NFPs or unconjugated

TABLE 1. Experimental animals for Vd/Vi ratio determination

\begin{tabular}{ccl}
\hline Group No. & No. of Animals & NFP \\
\hline 1 & 4 & NFP-100 \\
\hline 2 & 4 & NFP-400 \\
\hline 3 & 4 & NFP-1000 \\
\hline Control & 2 & Cy5.5 \\
\hline
\end{tabular}


TABLE 2. Experimental animals for NFP clearance determination

\begin{tabular}{cclc}
\hline Group No. & No. of Animals & \multicolumn{1}{c}{ NFP } & Killed (hrs) \\
\hline 1 & 4 & NFP-100 & 24 \\
\hline 2 & 4 & NFP-100 & 72 \\
\hline 3 & 4 & NFP-400 & 24 \\
\hline 4 & 4 & NFP-400 & 72 \\
\hline 5 & 4 & NFP-1000 & 24 \\
\hline 6 & 4 & NFP-1000 & 72 \\
\hline Control-A & 2 & Cy5.5 & 24 \\
\hline Control-B & 2 & Cy5.5 & 72 \\
\hline
\end{tabular}

Cy5.5 dissolved in PBS $(6 \mu \mathrm{l})$. Infusions were performed using a UMP-2 pump controlled by a Micro-4 pump controller (World Precision Instruments) at a rate of $0.1 \mu \mathrm{l} /$ min. At the completion of infusion, the needle was left in place for 5 minutes and then slowly withdrawn.

\section{Tissue Preparation and NFP Detection}

All experimental groups underwent intracardiac perfusion with $4 \%$ paraformaldehyde (PFA) under deep anesthesia. Once harvested, the brains were further fixed in $4 \% \mathrm{PFA}$ at $4^{\circ} \mathrm{C}$ for 48 hours before being processed and embedded in paraffin. Sections were cut at a thickness of $5 \mu \mathrm{m}$. Because control groups underwent infusion with unconjugated Cy5.5, after completion of CED the control animals were killed, and their brains were snap-frozen. After they were processed, frozen brains were cryosectioned at a thickness of $15 \mu \mathrm{m}$. All sections were mounted on slides, and NFP or Cy5.5 was detected using fluorescence microscopy (EVOS FL Cell Imaging System).

\section{Image Processing}

Fluorescence images were processed in Image $(\mathrm{NIH})$. $\mathrm{Vd}$ was determined using the ellipsoid method, ${ }^{27}$ where $\mathrm{Vd}=(\mathrm{ABC}) / 2$, and $\mathrm{A}$ is the greatest Vd diameter, $\mathrm{B}$ is the diameter $90^{\circ}$ to $\mathrm{A}$, and $\mathrm{C}$ is the approximate number of sections with NFP multiplied by the thickness of the section. Final volumes of distributions were multiplied by 1.6 to account for the approximately $60 \%$ shrinkage in brain volume after immersion in $4 \%$ PFA for 48 hours. ${ }^{53}$ Volume of distribution was divided by $\mathrm{Vi}$ to determine $\mathrm{Vd} / \mathrm{Vi}$ ratios. NFP clearance was determined by taking the calculated volume of the remaining NFP at 24 and 72 hours and dividing it by the Vi. All statistical analyses were performed using Stata (version IC 13.1, StataCorp LP).

\section{Results}

\section{NFP Design, Synthesis, and Characterization}

NFP was composed of multiple FITC-conjugated peptide constructs that self-assembled in an aqueous PBS buffer via electrostatic and hydrophobic interactions to form a single-layer NFP of approximately $5 \mathrm{~nm}$ in width (Fig. 1). ${ }^{28,29,34,52}$ The hydrophilic mPEG prevented individual NFPs from aggregation. ${ }^{24,55}$ The nanofiber surface was covalently conjugated with multiple Cy5.5 fluorophores as near-infrared optical reporters via the $-\mathrm{NH}_{2}$ groups on the lysine residues. The conjugation was successful, as con- firmed by ultraviolet absorbance (Fig. 1B). We calculated that there was approximately 1 molecule of Cy5.5 attached to 7 peptide constructs. NFPs were prepared in different sizes using a mini-extruder, as previously described, ${ }^{29}$ which resulted in NFPs of the same width but different lengths: $1000 \mathrm{~nm}$ (NFP-1000), $400 \mathrm{~nm}$ (NFP-400), and 100 nm (NFP-100). NFP morphology and size was characterized using TEM (Fig. 1D). NFPs were homogenous without signs of aggregation, with a negative surface charge (zeta potential $=-16 \mathrm{mV} \pm 2$ ). The luminescent cell viability assay performed on brain glial (astrocytes) and glioblastoma (U87, GS-9 L, and C6) cell lines revealed that NFP-100 was nontoxic even at high concentration (Fig. 1C).

\section{CED Procedure}

All NFPs were freshly prepared within 24 hours of CED and dissolved in PBS to final working concentrations of $20 \mathrm{nmol} / \mathrm{ml}$ of Cy5.5 content. CED of NFP was well tolerated by animals. There were no intraoperative deaths, and all animals awoke from anesthesia without any neurological symptoms. In animals killed 24 or 72 hours after CED, no delayed neurological complications were detected. Gait, feeding, excretion, and grooming were all normal following surgery and thereafter until the end of the study. Cranial nerves (corneal reflexes, whisker response, and symmetric facial movements) and motor and sensory functions of the body and extremities were also intact.

\section{NFP Volume of Distribution}

CED effectively distributed NFP in the striatum (Fig. 2). The average Vd/Vi ratios for NFP-100, NFP-400, NFP1000, and unconjugated Cy5.5 were 1.87 (range 1.652.12), 2.47 (range 2.20-2.60), 1.07 (range 0.81-1.18), and 3.0 (range 2.00-4.00), respectively, which were statistically different on 1-way ANOVA ( $\mathrm{p}=0.003)$. NFP distribution followed large white matter tracts, as indicated by the spread of NFP along the corpus callosum (Fig. 3). Lastly, all NFPs lined the subependymal surface and entered the choroid plexus and ventricular space (Fig. 3).

\section{NFP Clearance}

NFPs were detectable 24 and 72 hours after CED (Fig. 4A) while unconjugated Cy5.5 was undetectable at either time point. The percentages remaining of the original infusion volume at 24 hours for NFP-100, -400 , and -1000 were $40 \%$ (range $33 \%-61 \%$ ), $90 \%$ (range $78 \%-100 \%$ ), and $74 \%(72 \%-75 \%)$, respectively (Fig. 4B left). The percentages remaining at 72 hours for NFP-100, -400 , and -1000 were $15 \%$ (range $7.3 \%-28 \%$ ), $30 \%$ (range $22 \%-30 \%$ ), and $46 \%$ (range 34\%-54\%), respectively (Fig. 4B right).

\section{Discussion \\ CED of Nanocarriers}

Nanocarriers are drug vehicles that mainly include polymeric or lipid-based carriers, such as liposomes, nanoparticles, micelles, and dendrimers. ${ }^{2,5,12,26,38,39,45}$ Nanocarriers allow for improved drug delivery, reduced off-target effects, and improved drug kinetics. With nanocarriers, the pharmacokinetics of the drug no longer depend on the 

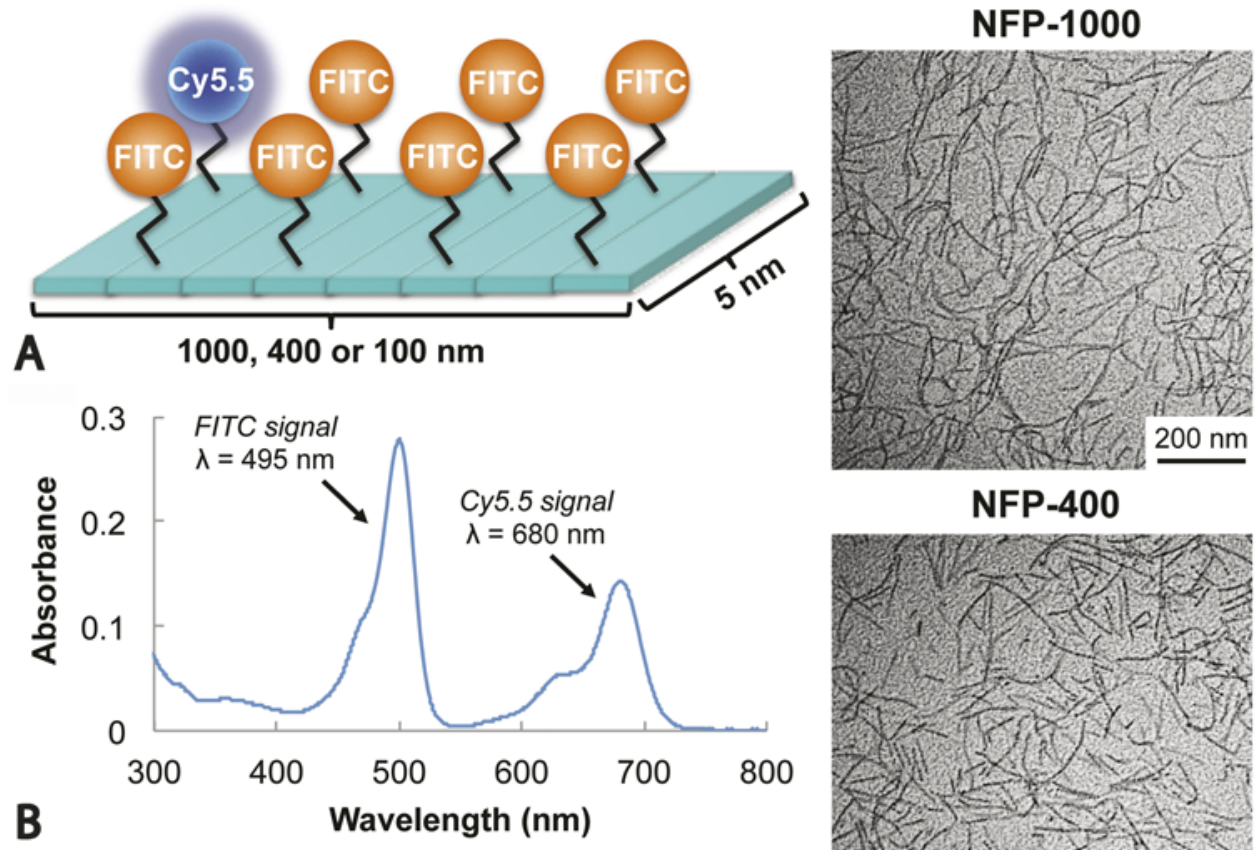

NFP-400
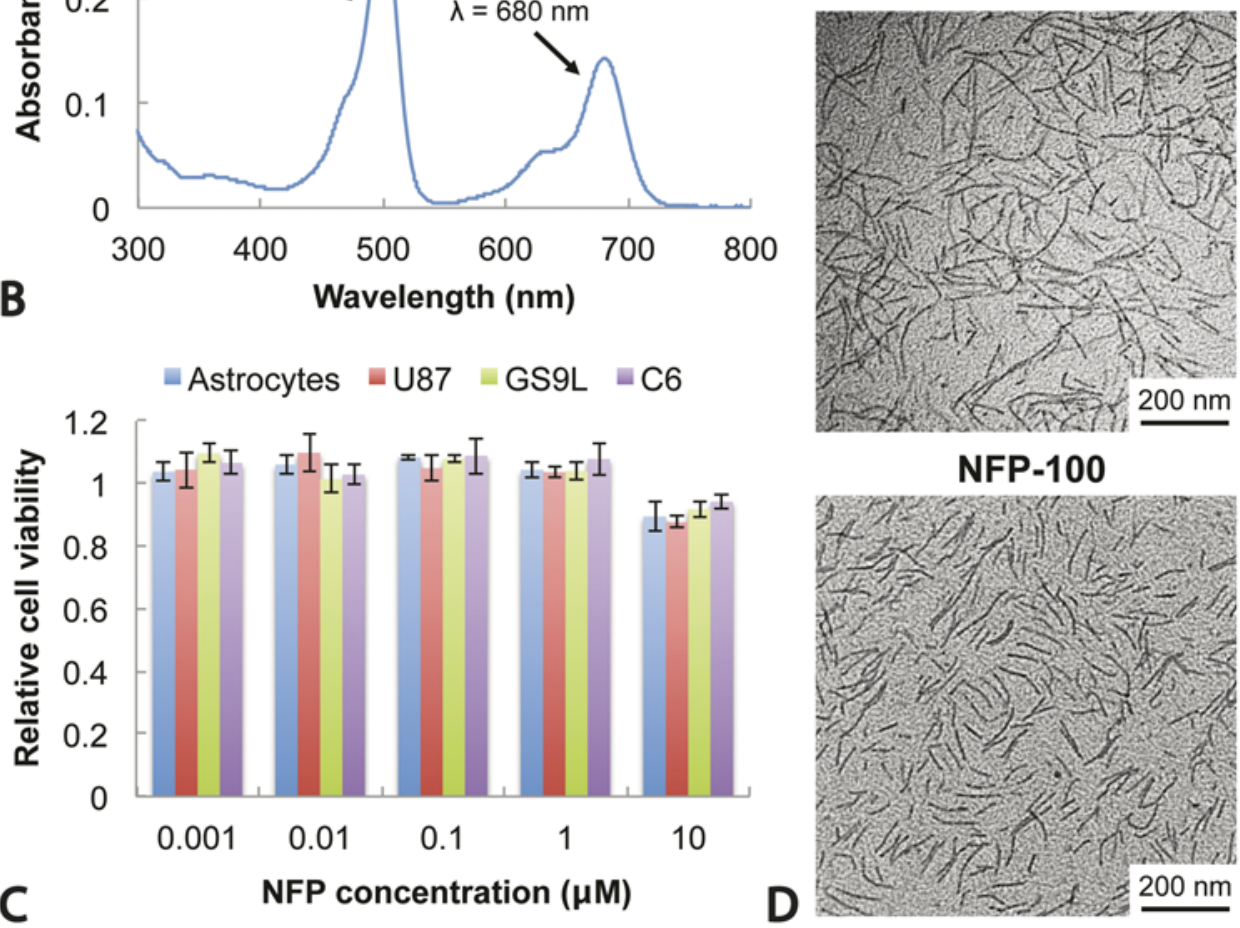

FIG. 1. NFP characteristics. A: Schematic representation of NFP assembled from FITC-conjugated peptide constructs. Cy5.5 fluorophores were conjugated on the nanofiber surface as near-infrared optical reporters. B: Ultraviolet absorption spectra confirming the conjugation of Cy5.5 fluorophores on the NFP surface. Measurements were performed in 5\% (v/v) PBS in methanol. The maximum absorptions $\left(\lambda_{\max }\right)$ of FITC and Cy5.5 were $495 \mathrm{~nm}$ and $680 \mathrm{~nm}$, respectively. C: Bar chart showing the cell viability of brain glial (astrocytes) and glioblastoma (U87, GS-9 L, and C6) cell lines after NFP exposure (1 nM to $10 \mu M$ of peptide content). All experiments were performed in triplicate. D: TEM images of NFP homogenized in different lengths: $1000 \mathrm{~nm}$ (NFP-1000), $400 \mathrm{~nm}$ (NFP-400), and $100 \mathrm{~nm}$ (NFP-100). All samples were stained with uranyl formate $(0.5 \% \mathrm{v} / \mathrm{v})$ prior to TEM analysis at a magnification of $\times 80,000$.

active molecule but rather on the physiochemical properties of the carrier. ${ }^{2}$ Each nanocarrier differs in structure composition, drug-loading capacity, ability to encapsulate hydrophobic or hydrophilic drugs, and carrier stability. Nanocarriers offer a diverse array of chemical possibilities, with the ability to carry multiple drugs, radionuclides, and imaging agents with target specificity. ${ }^{15,19,25,40}$ While liposomes have been widely used with CED, recently developed poly(lactic-co-glycolic acid) (PLGA) polymeric nanoparticles provide the advantage of long-term release.

Nanoparticle size is one of the most critical parameters for CED. $1,23,41,42,54,56$ CED delivers agents to the brain interstitium; therefore nanoparticle distribution and penetra- tion is limited by the size of the interstitial space, which is normally $38-64 \mathrm{~nm}$ but can vary from 7 to $100 \mathrm{~nm}$ in tumors. ${ }^{22,50} \mathrm{The} \mathrm{Vd} / \mathrm{Vi}$ ratio, a marker of drug distribution, decreases as nanocarrier size increases, with the preference for nanocarriers approximately $70 \mathrm{~nm}$ or less in size for CED. Previous studies have reported an approximately 2-8-fold increase in $\mathrm{Vd} / \mathrm{Vi}$ ratios for approximately 70 $\mathrm{nm}$ nanoparticles when compared with approximately 150 -nm nanoparticles. ${ }^{10,46,49,56}$

\section{Advantages of NFP}

While recent advances in nanoparticle design allow for PLGA nanoparticles approximately $70 \mathrm{~nm}$ in size, the 

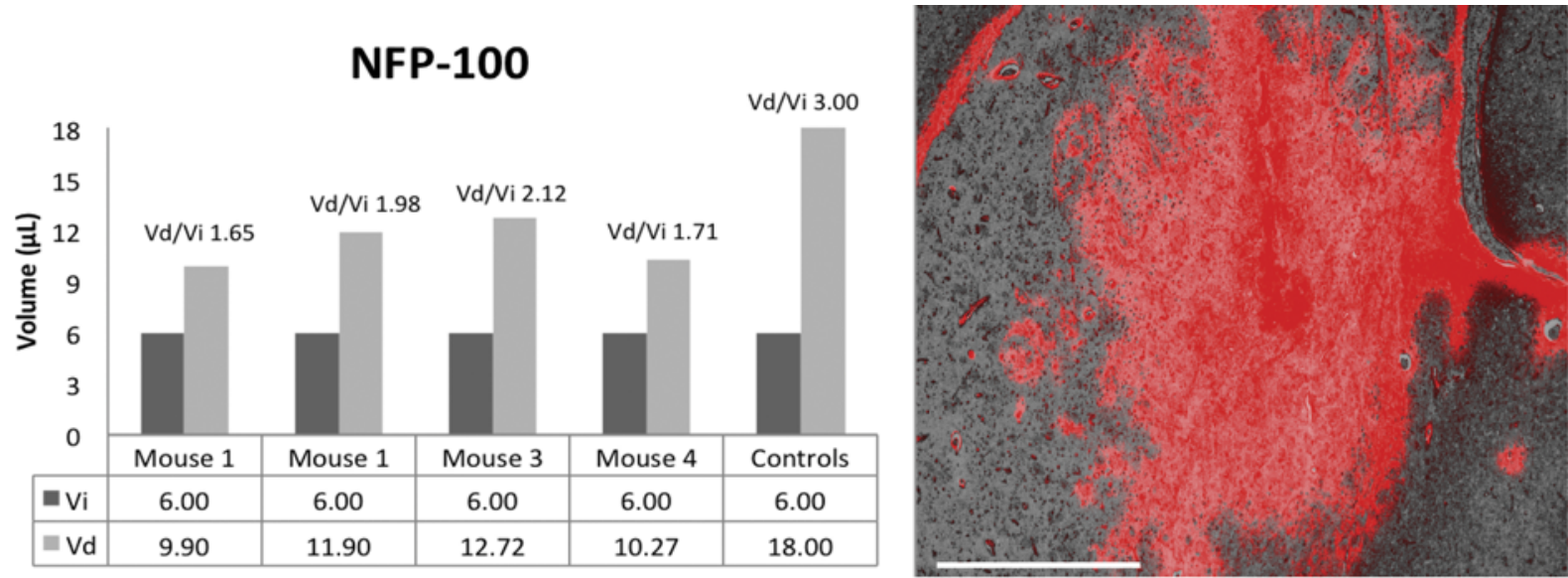

\section{NFP-400}
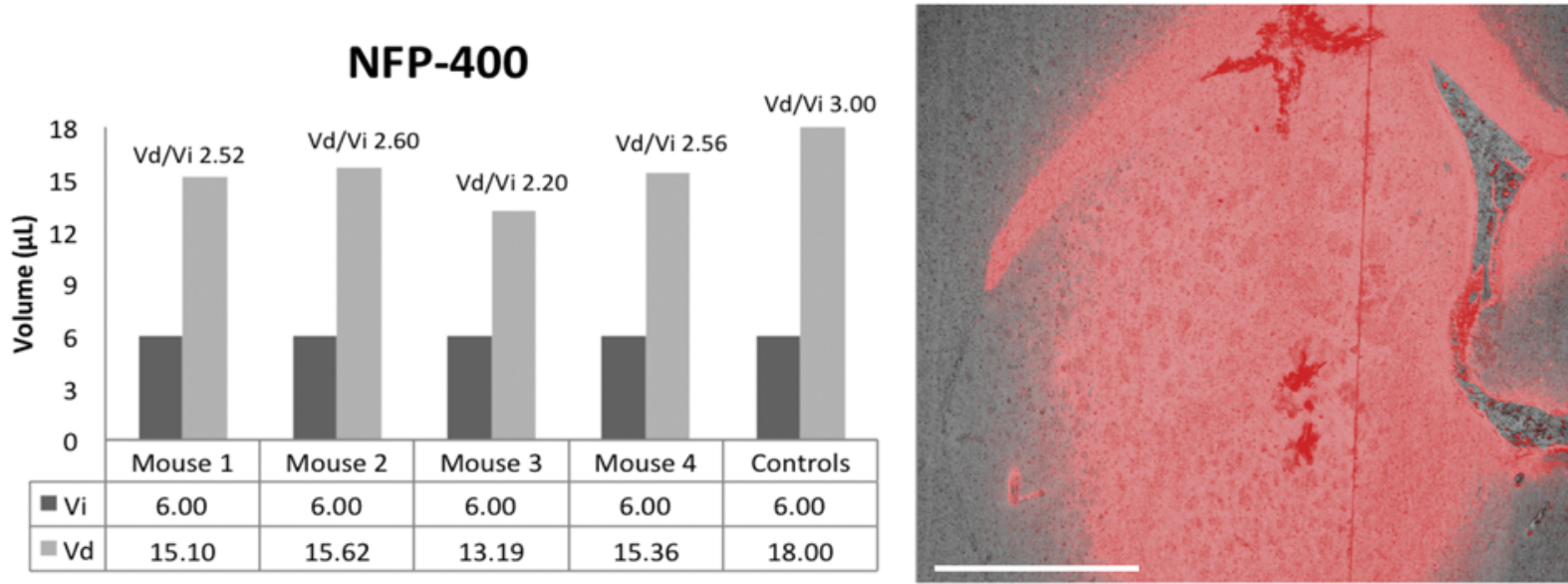

NFP-1000
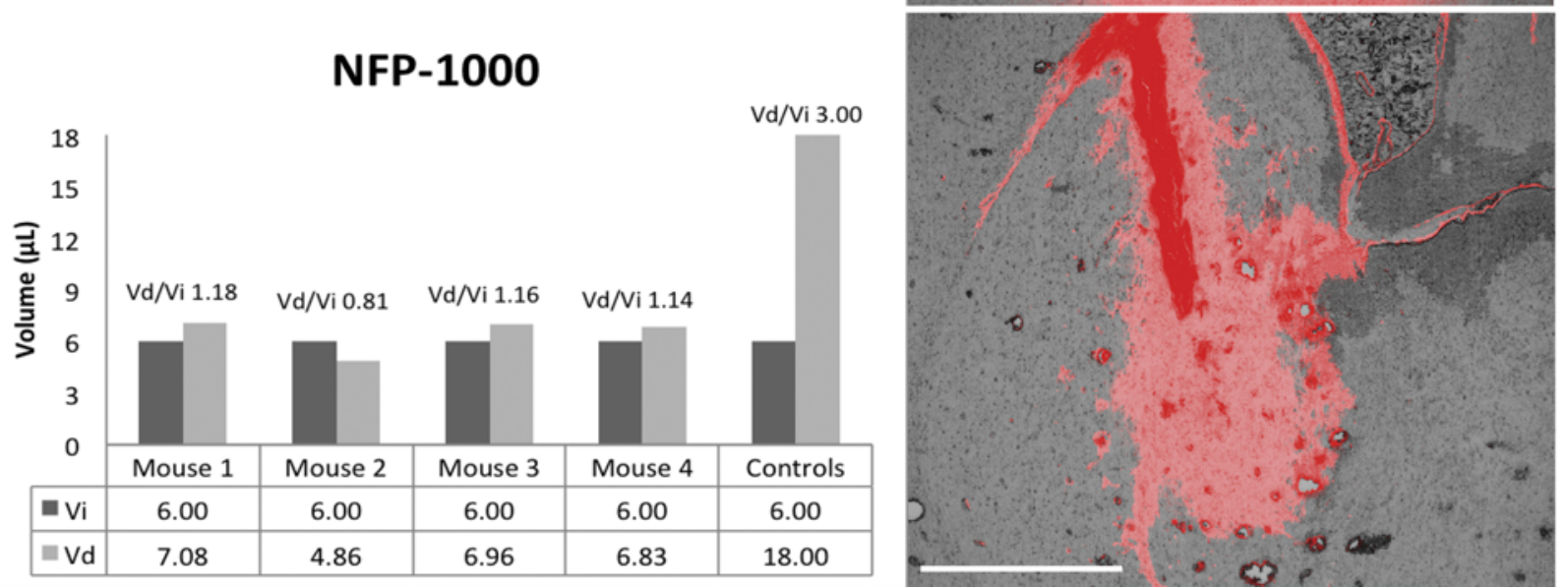

FIG. 2. NFPs of different lengths show distinctive brain distributions after CED. Comparison of the Vd/Vi ratios of NFP-100, -400 , and -1000 (left column), with corresponding sections through the cannula tract of NFP distribution in mouse striatum (right column; bar $=1000 \mu \mathrm{m}$, magnification $\times 40$ ). Two control animals were infused with unconjugated Cy5.5, and the average $\mathrm{Vd} / \mathrm{Vi}$ is reported.

issue of size continues to present a challenge in CED of nanoparticles. First, not all nanoparticles in a synthesis are homogenous. In current reported PLGA nanoparticle synthesis, the size of the nanoparticles can vary by as much as $18-30 \mathrm{~nm}$, and a slight change in size could alter the nanoparticle Vd. ${ }^{56}$ Second, most experiments that reported increased $\mathrm{Vd} / \mathrm{Vi}$ ratios using 70 -nm nanoparticles were performed in naïve animals. Due to the size heterogeneity of tumor interstitial space, ${ }^{22}$ a 70 -nm nanoparticle may attain appropriate $\mathrm{Vd}$ in some tumor regions but not in others. One study reported $\mathrm{Vd} / \mathrm{Vi}$ ratios of $<1$ when nanoparticles approximately $70 \mathrm{~nm}$ in size were delivered via CED. ${ }^{10}$ Inadequate Vd could lead to suboptimal tumor coverage by drug and failed therapy. ${ }^{44,51}$

NFPs offer design flexibility that could potentially enable better navigation to and extravasation of tumor tissue than is possible with rigid nanotubes or nanoparticles. Unlike other drug nanofibers, NFPs do not form 

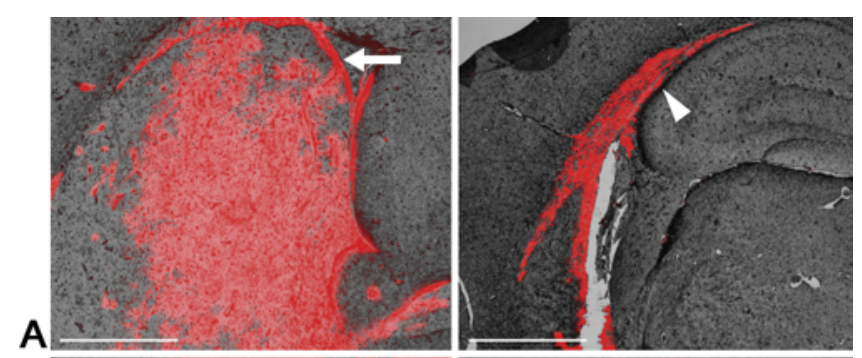

B
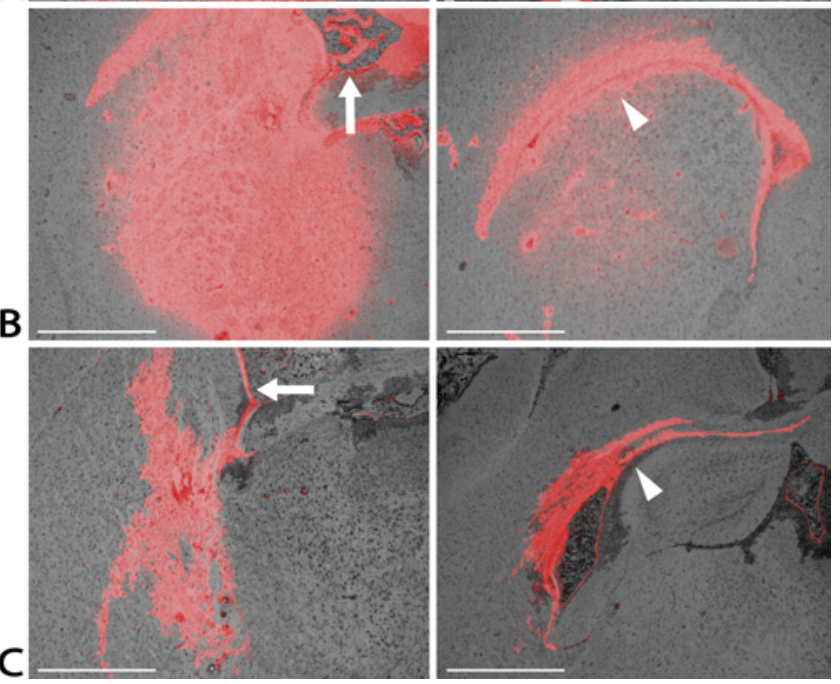

C

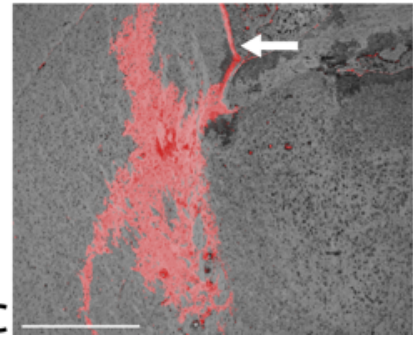

FIG. 3. NFP distribution along white matter tracts and subependymal surfaces. A: NFP-100 distribution in the striatum and along the subependymal surface of the lateral ventricle (arrow) and corpus callosum (arrowhead). B: NFP-400 distribution in the striatum and along the subependymal surface and in the intraventricular space of the lateral ventricle (arrow) and corpus callosum (arrowhead). C: NFP-1000 distribution in the striatum and along the subependymal surface (arrow) and the corpus callosum (arrowhead). Sections highlighting the distribution along the corpus callosum were taken $4-5$ sections away from the cannula tip. Bar $=1000 \mu \mathrm{m}$, magnification $\times 40$.

cylindrical nanotubes and thus retain their single-layer $2 \mathrm{D}$ shape. ${ }^{28,29,34,52}$ Furthermore, the NFP surface is negatively charged and contains hydrophilic mPEG, both design features that reduce nanocarrier interaction with extracellular matrix proteins and promote larger distribution. Studies have shown that cationic carriers stay adjacent to the cannula tract due to nonspecific interactions with negatively charged protein in brain parenchyma, and these interactions can be minimized with pegylation, making a negatively charged outer surface with supporting pegylation desirable to maximize distribution..$^{33,43}$

The design flexibility of NFP, negative surface charge, and pegylation may allow for therapeutic $\mathrm{Vd} / \mathrm{Vi}$ ratios in tumors despite heterogeneity in the size and properties of tumor interstitial space. In the current study, we tested the Vd and clearance of NFP-100, NFP-400, and NFP-1000.

\section{NFP Distribution After CED}

The average Vd/Vi ratios for NFP-100, NFP-400, NFP1000 , and unconjugated Cy5.5 were 1.87, 2.47, 1.07, and 3.0 , respectively. These $\mathrm{Vd} / \mathrm{Vi}$ ratios are comparable with those reported in prior published results from other nanocarriers, which have ranged between 1 and 5.5.2,10,38,46,49,56
NFP-400 outperformed the other NFPs for Vd. Several factors could account for the higher Vd/Vi ratio of NFP400. NFP-1000 mostly stayed around the cannula tract in the ventral-dorsal plane, likely a result of its large size, and was therefore unable to adequately move through the interstitial space. NFP-100 had prominent spread along white matter tracts and into the intraventricular space. Due to the relatively small size of NFP-100, it is plausible that rather than staying localized, NFP-100 followed white matter tracts and was cleared into the intraventricular space. NFP400 appeared to have a clinically useful $\mathrm{Vd} / \mathrm{Vi}$ ratio with a fairly spherical distribution from the cannula tip. NFP400 size was adequate to obtain a clinically applicable $\mathrm{Vd} /$ $\mathrm{Vi}$ ratio but also large enough to minimize entry into the intraventricular space and spread along white matter tracts.

\section{NFP Clearance}

The infusate continues to distribute, both via natural bulk flow and diffusion, after CED. This is especially the case for small molecules and may lead to quick decline in drug concentration after CED. Modification of drug pharmacokinetics is one of the many advantages of using nanocarriers. Compared with unconjugated Cy5.5, a significant amount of NFP was observable at 24 and 72 hours after CED. This result is consistent with previous studies that report rapid clearance of unbound ligands compared with ligands bound to nanocarriers. ${ }^{46}$

Published half-lives (i.e., the time to clear half of the infusate) of nanocarriers delivered via CED have ranged from a few minutes to approximately 10 hours. ${ }^{1,2,33,46,48}$ Compared with these values, NFPs appear to outperform other nanocarriers with slower clearance rates. As expected, NFP-100 was rapidly cleared from the interstitial space, yet $40 \%$ was still observable at 24 hours, with a calculated half-life of approximately 18 hours. Interestingly, nearly the entire NFP-400 infusate volume was detectable at 24 hours after infusion, whereas $30 \%$ was observable at 72 hours, with a calculated half-life of approximately 25 hours. Nearly $74 \%$ of NFP-1000 was observable at 24 hours and $46 \%$ at 72 hours, with a calculated half-life of approximately 42 hours. Therefore, while NFP-1000 had a low $\mathrm{Vd}$, a large percentage of it remained in the interstitium for an extended period after CED.

Clearance of NFP from the interstitial space could occur by 2 potential pathways: 1) Cy5.5 is liberated from the NFP surface and cleared while the NFP still remains in the interstitial space; or 2) NFP with attached Cy5.5 is cleared from the interstitial space. Because the NFP surface is covalently conjugated with $\mathrm{Cy} 5.5$ via the $-\mathrm{NH}_{2}$ group of the lysine residue, it is highly unlikely that Cy5.5 would be liberated from the NFP surface and cleared independently. Furthermore, prior studies from our group have shown the Cy5.5 conjugation to be stable and able to withstand biodistribution. ${ }^{52}$ Therefore, it is likely that the NFP with attached Cy5.5 is cleared from the interstitial space.

\section{Limitations of the Study}

One limitation of this study is that there was no reliable quantitative way to convert fluorescence signal into concentration. Therefore, while we studied the Vd and clearance using calculated volumes, determining the concentra- 


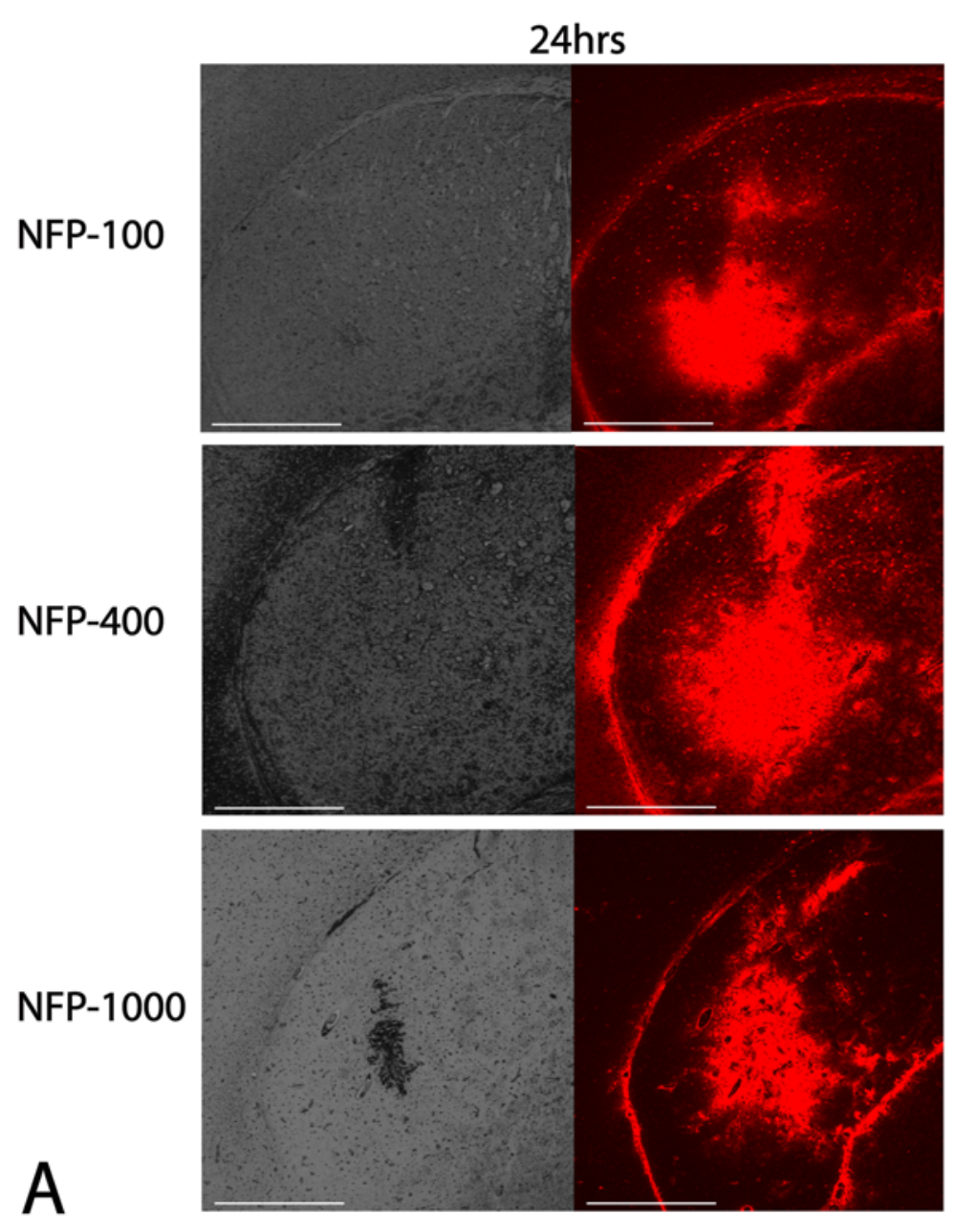

NFP at 24hrs post CED

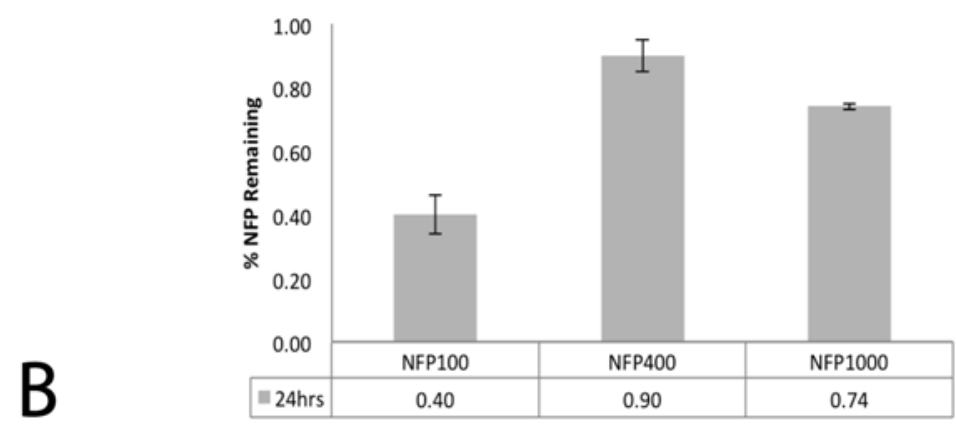

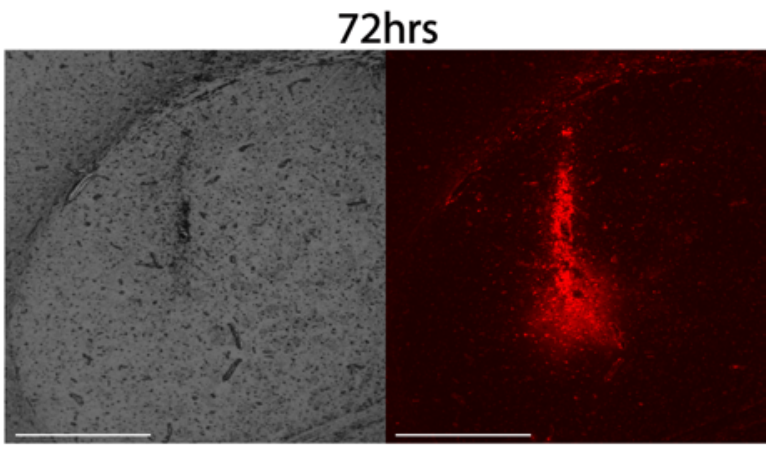
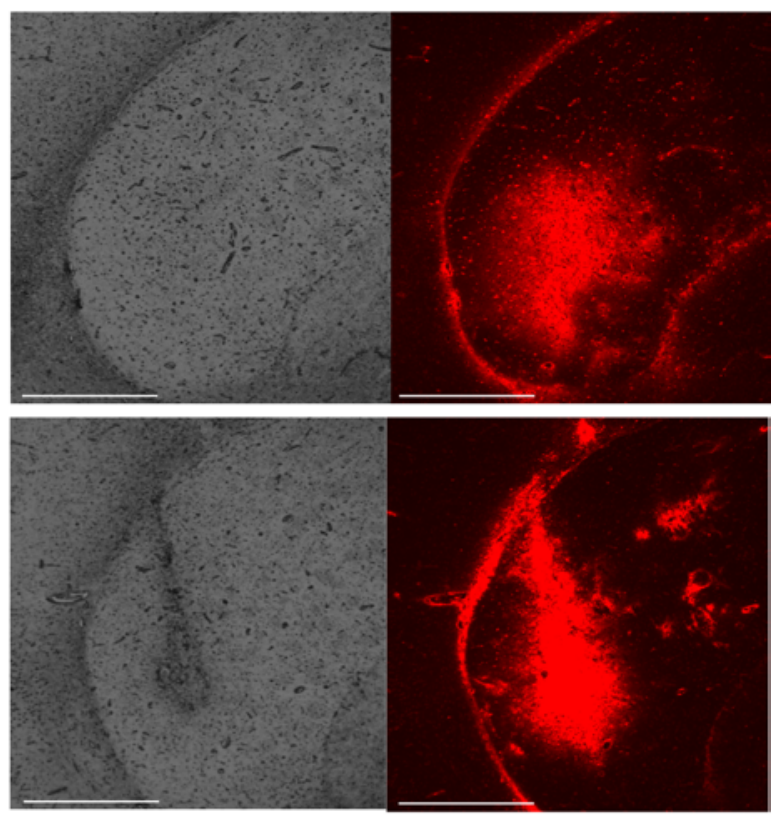

NFP at $72 \mathrm{hrs}$ post CED

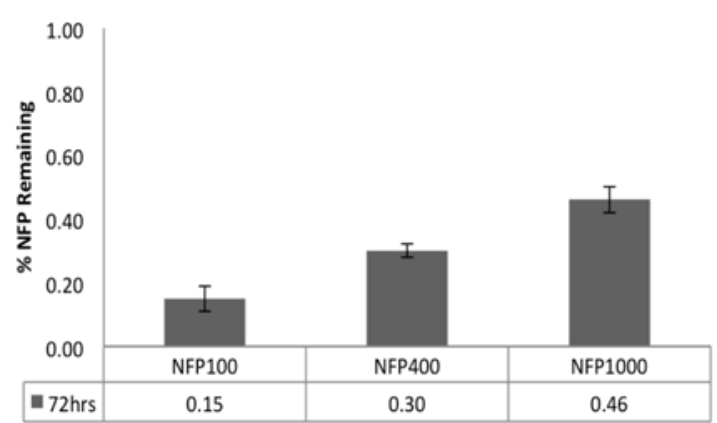

FIG. 4. A comparison of NFP clearance with time. A: Sections through the cannula tract for NFP-100, -400, and -1000 24 hours (left column) and 72 hours (right column) after CED. Fluorescent images are provided along with their corresponding transmitted light images. Bar $=1000 \mu \mathrm{m}$, magnification $\times 40$. B: Percentage of NFP remaining at 24 and 72 hours after CED.

tion of the agent using fluorescence signal was not feasible. Concentration determination in vivo is critical in CED and is a matter of active research at the moment. One potential way to determine NFP concentration in vivo is to radiolabel the NFP and use radioactive signal, which our group is currently pursuing. Dual-labeled NFP with a radioisotope and fluorophore would allow for both in vivo and ex vivo monitoring of drug distribution and concentration. Certain features of the physical properties of NFP infusion via CED still need to be clarified. Namely, optimizing the rate of infusion, appropriate cannula design, Vd of NFP in different regions of the brain, affinity of the brain extracellular matrix for NFP, ways that Vd can be enhanced and clearance can be slowed, and long-term toxicity studies of NFP after CED.

\section{Conclusions}

NFP has the potential for wide use and applicability in CED for brain tumor treatment. NFP provides design flex- 
ibility, clinically useful Vd/Vi ratios, and desirable slow clearance rates from the brain interstitium. NFP therefore has the potential to maintain effective drug concentrations for an extended period sufficient for therapy without the need for prolonged infusions or reinfusions. Specific ligands, such as chemotherapeutic small molecules, antibodies, and radioisotopes, can be conjugated onto the NFP surface, allowing for targeted drug therapy, improved drug pharmacokinetics, and in vivo drug monitoring. The prolonged retention time of NFP in the brain will allow us to control the drug release kinetics. Previously, we designed NFP analogs that can be digested by lysosomal enzymes to release the drug content. ${ }^{9}$ In the future we will incorporate conventional chemotherapeutic and other molecularly targeted agents (e.g., panobinostat ${ }^{17}$ ) to NFP via various environmentally sensitive linkers ( $\mathrm{pH}$ or enzyme) to control the release kinetics. Furthermore, NFP can be optimized for delivery to different tumor types. For example, NFP-100 may be best suited for tumors that grow along white matter tracts, such as DIPGs. NFP-1000 may be best suited for tumors that require extended therapy, such as low-grade gliomas, for which inadequate therapy may promote malignant transformation. NFP-400 may work best for larger tumors that require both large $\mathrm{Vd}$ and extended therapy, such as glioblastoma. NFP provides a dynamic theranostic platform, with the potential to deliver clinically efficacious drug payload to brain tumors after CED.

\section{Acknowledgments}

Funding for this study was provided to Dr. Singh by the St. Baldrick's Summer Fellowship, St. Baldrick's Foundation; the Paul Calabresi Medical Student Fellowship, PhRMA Foundation; and the Carolyn L. Kuckein Student Research Fellowship, Alpha Omega Alpha.

\section{References}

1. Allard E, Hindre F, Passirani C, Lemaire L, Lepareur N, Noiret N, et al: ${ }^{188} \mathrm{Re}$-loaded lipid nanocapsules as a promising radiopharmaceutical carrier for internal radiotherapy of malignant gliomas. Eur J Nucl Med Mol Imaging 35:18381846,2008

2. Allard E, Passirani C, Benoit JP: Convection-enhanced delivery of nanocarriers for the treatment of brain tumors. Biomaterials 30:2302-2318, 2009

3. Anderson RC, Kennedy B, Yanes CL, Garvin J, Needle M, Canoll P, et al: Convection-enhanced delivery of topotecan into diffuse intrinsic brainstem tumors in children. J Neurosurg Pediatr 11:289-295, 2013

4. Asthagiri AR, Walbridge S, Heiss JD, Lonser RR: Effect of concentration on the accuracy of convective imaging distribution of a gadolinium-based surrogate tracer. J Neurosurg 115:467-473, 2011

5. Barbu E, Molnàr E, Tsibouklis J, Górecki DC: The potential for nanoparticle-based drug delivery to the brain: overcoming the blood-brain barrier. Expert Opin Drug Deliv 6:553565,2009

6. Barua NU, Hopkins K, Woolley M, O’Sullivan S, Harrison R, Edwards RJ, et al: A novel implantable catheter system with transcutaneous port for intermittent convection-enhanced delivery of carboplatin for recurrent glioblastoma. Drug Deliv 23:167-173, 2016

7. Barua NU, Lowis SP, Woolley M, O’Sullivan S, Harrison R, Gill SS: Robot-guided convection-enhanced delivery of carboplatin for advanced brainstem glioma. Acta Neurochir (Wien) 155:1459-1465, 2013
8. Becher OJ, Hambardzumyan D, Walker TR, Helmy K, Nazarian J, Albrecht S, et al: Preclinical evaluation of radiation and perifosine in a genetically and histologically accurate model of brainstem glioma. Cancer Res 70:2548-2557, 2010

9. Bellat V, Lee HH, Vahdat L, Law B: Smart nanotransformers with unique enzyme-inducible structural changes and drug release properties. Biomacromolecules 17:2040-2049, 2016

10. Bernal GM, LaRiviere MJ, Mansour N, Pytel P, Cahill KE, Voce DJ, et al: Convection-enhanced delivery and in vivo imaging of polymeric nanoparticles for the treatment of malignant glioma. Nanomedicine (Lond) 10:149-157, 2014

11. Bienemann A, White E, Woolley M, Castrique E, Johnson DE, Wyatt M, et al: The development of an implantable catheter system for chronic or intermittent convection-enhanced delivery. J Neurosci Methods 203:284-291, 2012

12. Blanco E, Hsiao A, Mann AP, Landry MG, Meric-Bernstam F, Ferrari M: Nanomedicine in cancer therapy: innovative trends and prospects. Cancer Sci 102:1247-1252, 2011

13. Bobo RH, Laske DW, Akbasak A, Morrison PF, Dedrick RL, Oldfield EH: Convection-enhanced delivery of macromolecules in the brain. Proc Natl Acad Sci U S A 91:2076-2080, 1994

14. Boiardi A, Silvani A, Eoli M, Lamperti E, Salmaggi A, Gaviani $P$, et al: Treatment of recurrent glioblastoma: can local delivery of mitoxantrone improve survival? J Neurooncol 88:105-113, 2008

15. Cho K, Wang X, Nie S, Chen ZG, Shin DM: Therapeutic nanoparticles for drug delivery in cancer. Clin Cancer Res 14:1310-1316, 2008

16. Croteau D, Walbridge S, Morrison PF, Butman JA, Vortmeyer AO, Johnson D, et al: Real-time in vivo imaging of the convective distribution of a low-molecular-weight tracer. J Neurosurg 102:90-97, 2005

17. Grasso CS, Tang Y, Truffaux N, Berlow NE, Liu L, Debily MA, et al: Functionally defined therapeutic targets in diffuse intrinsic pontine glioma. Nat Med 21:555-559, 2015

18. Groothuis DR, Ward S, Itskovich AC, Dobrescu C, Allen CV, Dills C, et al: Comparison of ${ }^{14} \mathrm{C}$-sucrose delivery to the brain by intravenous, intraventricular, and convection-enhanced intracerebral infusion. J Neurosurg 90:321-331, 1999

19. Hamoudeh M, Kamleh MA, Diab R, Fessi H: Radionuclides delivery systems for nuclear imaging and radiotherapy of cancer. Adv Drug Deliv Rev 60:1329-1346, 2008

20. Hargrave D, Bartels U, Bouffet E: Diffuse brainstem glioma in children: critical review of clinical trials. Lancet Oncol 7:241-248, 2006

21. Ho SL, Singh R, Zhou Z, Lavi E, Souweidane MM: Toxicity evaluation of prolonged convection-enhanced delivery of small-molecule kinase inhibitors in naïve rat brainstem. Childs Nerv Syst 31:221-226, 2015

22. Hobbs SK, Monsky WL, Yuan F, Roberts WG, Griffith L, Torchilin VP, et al: Regulation of transport pathways in tumor vessels: role of tumor type and microenvironment. Proc Natl Acad Sci U S A 95:4607-4612, 1998

23. Inoue T, Yamashita Y, Nishihara M, Sugiyama S, Sonoda Y, Kumabe T, et al: Therapeutic efficacy of a polymeric micellar doxorubicin infused by convection-enhanced delivery against intracranial 9L brain tumor models. Neuro Oncol 11:151-157, 2009

24. Kisiday J, Jin M, Kurz B, Hung H, Semino C, Zhang S, et al: Self-assembling peptide hydrogel fosters chondrocyte extracellular matrix production and cell division: implications for cartilage tissue repair. Proc Natl Acad Sci U S A 99:999610001, 2002

25. Kobayashi H, Brechbiel MW: Dendrimer-based nanosized MRI contrast agents. Curr Pharm Biotechnol 5:539-549, 2004

26. Koo YE, Reddy GR, Bhojani M, Schneider R, Philbert MA, Rehemtulla A, et al: Brain cancer diagnosis and therapy with nanoplatforms. Adv Drug Deliv Rev 58:1556-1577, 2006 
27. Kothari RU, Brott T, Broderick JP, Barsan WG, Sauerbeck LR, Zuccarello M, et al: The ABCs of measuring intracerebral hemorrhage volumes. Stroke 27:1304-1305, 1996

28. Law B, Tung CH: Structural modification of protease inducible preprogrammed nanofiber precursor. Biomacromolecules 9:421-425, 2008

29. Law B, Weissleder R, Tung CH: Protease-sensitive fluorescent nanofibers. Bioconjug Chem 18:1701-1704, 2007

30. Lonser RR, Sarntinoranont M, Morrison PF, Oldfield EH: Convection-enhanced delivery to the central nervous system. J Neurosurg 122:697-706, 2015

31. Lonser RR, Warren KE, Butman JA, Quezado Z, Robison RA, Walbridge S, et al: Real-time image-guided direct convective perfusion of intrinsic brainstem lesions. Technical note. J Neurosurg 107:190-197, 2007

32. Lopez KA, Tannenbaum AM, Assanah MC, Linskey K, Yun J, Kangarlu A, et al: Convection-enhanced delivery of topotecan into a PDGF-driven model of glioblastoma prolongs survival and ablates both tumor-initiating cells and recruited glial progenitors. Cancer Res 71:3963-3971, 2011

33. MacKay JA, Deen DF, Szoka FC Jr: Distribution in brain of liposomes after convection enhanced delivery; modulation by particle charge, particle diameter, and presence of steric coating. Brain Res 1035:139-153, 2005

34. Malik R, Qian S, Law B: Design and synthesis of a nearinfrared fluorescent nanofiber precursor for detecting cellsecreted urokinase activity. Anal Biochem 412:26-33, 2011

35. Occhiogrosso G, Edgar MA, Sandberg DI, Souweidane MM: Prolonged convection-enhanced delivery into the rat brainstem. Neurosurgery 52:388-394, 2003

36. Park KC, Toyonaga S, Nakabayashi H, Mizobuchi H, Nakai E, Ikawa N, et al: Primitive neuroectodermal tumor (PNET) treated with interferon- $\beta$ after surgical removal and irradiation: case report. Anticancer Res 24 (2C):1105-1110, 2004

37. Patchell RA, Regine WF, Ashton P, Tibbs PA, Wilson D, Shappley D, et al: A phase I trial of continuously infused intratumoral bleomycin for the treatment of recurrent glioblastoma multiforme. J Neurooncol 60:37-42, 2002

38. Patel T, Zhou J, Piepmeier JM, Saltzman WM: Polymeric nanoparticles for drug delivery to the central nervous system. Adv Drug Deliv Rev 64:701-705, 2012

39. Patel TR: Nanocarrier-based therapies for CNS tumors. CNS Oncol 3:115-122, 2014

40. Peer D, Karp JM, Hong S, Farokhzad OC, Margalit R, Langer R: Nanocarriers as an emerging platform for cancer therapy. Nat Nanotechnol 2:751-760, 2007

41. Perlstein B, Ram Z, Daniels D, Ocherashvilli A, Roth Y, Margel S, et al: Convection-enhanced delivery of maghemite nanoparticles: Increased efficacy and MRI monitoring. Neuro Oncol 10:153-161, 2008

42. Saito R, Bringas JR, McKnight TR, Wendland MF, Mamot C, Drummond DC, et al: Distribution of liposomes into brain and rat brain tumor models by convection-enhanced delivery monitored with magnetic resonance imaging. Cancer Res 64:2572-2579, 2004

43. Saito R, Krauze MT, Noble CO, Tamas M, Drummond DC, Kirpotin DB, et al: Tissue affinity of the infusate affects the distribution volume during convection-enhanced delivery into rodent brains: implications for local drug delivery. J Neurosci Methods 154:225-232, 2006

44. Sampson JH, Archer G, Pedain C, Wembacher-Schröder E, Westphal M, Kunwar S, et al: Poor drug distribution as a possible explanation for the results of the PRECISE trial. J Neurosurg 113:301-309, 2010

45. Sawyer AJ, Piepmeier JM, Saltzman WM: New methods for direct delivery of chemotherapy for treating brain tumors. Yale J Biol Med 79:141-152, 2006

46. Sirianni RW, Zheng MQ, Patel TR, Shafbauer T, Zhou J, Saltzman WM, et al: Radiolabeling of poly(lactic-co-glycolic acid) (PLGA) nanoparticles with biotinylated F-18 prosthetic groups and imaging of their delivery to the brain with positron emission tomography. Bioconjug Chem 25:2157-2165, 2014

47. Sonabend AM, Stuart RM, Yun J, Yanagihara T, Mohajed $\mathrm{H}$, Dashnaw S, et al: Prolonged intracerebral convection-enhanced delivery of topotecan with a subcutaneously implantable infusion pump. Neuro Oncol 13:886-893, 2011

48. Stephen ZR, Kievit FM, Veiseh O, Chiarelli PA, Fang C, Wang K, et al: Redox-responsive magnetic nanoparticle for targeted convection-enhanced delivery of O6-benzylguanine to brain tumors. ACS Nano 8:10383-10395, 2014

49. Strohbehn G, Coman D, Han L, Ragheb RR, Fahmy TM, Huttner AJ, et al: Imaging the delivery of brain-penetrating PLGA nanoparticles in the brain using magnetic resonance. J Neurooncol 121:441-449, 2015

50. Thorne RG, Nicholson C: In vivo diffusion analysis with quantum dots and dextrans predicts the width of brain extracellular space. Proc Natl Acad Sci U S A 103:5567-5572, 2006

51. Vavra M, Ali MJ, Kang EW, Navalitloha Y, Ebert A, Allen $\mathrm{CV}$, et al: Comparative pharmacokinetics of $14 \mathrm{C}$-sucrose in RG-2 rat gliomas after intravenous and convection-enhanced delivery. Neuro Oncol 6:104-112, 2004

52. Wagh A, Singh J, Qian S, Law B: A short circulating peptide nanofiber as a carrier for tumoral delivery. Nanomedicine (Lond) 9:449-457, 2013

53. Wehrl HF, Bezrukov I, Wiehr S, Lehnhoff M, Fuchs K, Mannheim JG, et al: Assessment of murine brain tissue shrinkage caused by different histological fixatives using magnetic resonance and computed tomography imaging. Histol Histopathol 30:601-613, 2015

54. Wu G, Barth RF, Yang W, Kawabata S, Zhang L, GreenChurch K: Targeted delivery of methotrexate to epidermal growth factor receptor-positive brain tumors by means of cetuximab (IMC-C225) dendrimer bioconjugates. Mol Cancer Ther 5:52-59, 2006

55. Zhang S: Fabrication of novel biomaterials through molecular self-assembly. Nat Biotechnol 21:1171-1178, 2003

56. Zhou J, Patel TR, Sirianni RW, Strohbehn G, Zheng MQ, Duong N, et al: Highly penetrative, drug-loaded nanocarriers improve treatment of glioblastoma. Proc Natl Acad Sci U S A 110:11751-11756, 2013

57. Zhou Z, Ho SL, Singh R, Pisapia DJ, Souweidane MM: Toxicity evaluation of convection-enhanced delivery of smallmolecule kinase inhibitors in naïve mouse brainstem. Childs Nerv Syst 31:557-562, 2015

\section{Disclosures}

Dr. Souweidane reports being a consultant to Aesculap.

\section{Author Contributions}

Conception and design: Law, Singh, Bellat, Souweidane. Acquisition of data: Singh, Bellat, Wang, Schweitzer, Wu. Analysis and interpretation of data: Law, Singh, Bellat, Wang, Schweitzer, Wu, Souweidane. Drafting the article: all authors. Critically revising the article: Law, Singh, Bellat, Tung, Souweidane. Reviewed submitted version of manuscript: Law, Singh, Souweidane. Approved the final version of the manuscript on behalf of all authors: Law. Statistical analysis: Singh. Administrative/technical/material support: Schweitzer, Wu, Tung, Souweidane. Study supervision: Law, Tung, Souweidane.

\section{Correspondence}

Benedict Law, Molecular Imaging Innovations Institute, Department of Radiology, Weill Cornell Medicine, 413 East 69th St., New York, NY 10021. email: sbl2004@med.cornell.edu. 